\title{
HARMONIC TRANSFORMATION THEORY OF ISOTHERMAL FAMILIES
}

\author{
EDWARD KASNER AND JOHN DECICCO
}

\section{Harmonic transformations. Let a transformation $T$}

$$
X+\phi(x, y), \quad Y=\psi(x, y),
$$

with the jacobian

$$
J=\phi_{x} \psi_{y}-\phi_{y} \psi_{x} \neq 0,
$$

be such that the components $\phi$ and $\psi$ satisfy the Laplace equation

$$
\phi_{x x}+\phi_{y y}=0, \quad \psi_{x x}+\psi_{y y}=0,
$$

in a certain region of the real (or complex) cartesian plane. We shall term any such correspondence $T$ a harmonic transformation.

The harmonic transformations form an infinite set $(H)$ of $\infty^{4 f(1)}$ correspondences since they are defined essentially by four independent functions of a single variable. The totality of harmonic correspondences of course do not constitute a group. ${ }^{1}$

A subset of the class $(H)$ of harmonic transformations is the conformal group. The components $\phi$ and $\psi$ of a conformal map are of course conjugate-harmonic, that is, they satisfy the direct or reverse Cauchy-Riemann equations

$$
\phi_{x}= \pm \psi_{y}, \quad \phi_{y}=\mp \psi_{x} .
$$

Thus a harmonic transformation is conformal if and only if its components are conjugate-harmonic. In general, the components of a harmonic transformation are not interrelated in any way whatsoever.

We have proved that in the real domain the only groups contained in the infinite set $(H)$ of $\infty^{4 f(1)}$ harmonic transformations are the group of $\infty^{2 f(1)}$ conformal maps, the group of $\infty^{6}$ affinities, and the subgroups of these two. In the imaginary domain, we have found in addition two extra infinite groups each consisting of $\infty^{1+2 f(1)}$ transformations.

Presented to the Society, November 2, 1946, under the title Geometry of harmonic transformations; received by the editors November 8, 1946 .

${ }_{1}^{1}$ Harmonic functions and harmonic transformations appear in the theory of minimal surfaces and the Plateau problem. In particular see the fundamental papers of Schwarz and Douglas. Schwarz discusses the case where the jacobian of a harmonic transformation vanishes and studies possible singularities. See Abhandlungen, vol. 1, p. 293. Douglas studies the inverse of harmonic transformations. See abstracts in Bull. Amer. Math. Soc. vol. 49 (1943) and vol. 50 (1944). 
In the present paper, we shall study the isothermal properties of harmonic transformations. We obtain characterizations not only of our set $(H)$ of harmonic correspondences but also new characterizations of the conformal group. See our Theorems 1, 2, 3, 4 and 5.

2. Minimal coordinates. In order to derive our results simply, it is found advantageous to introduce the minimal coordinates $u=x+i y$, $v=x-i y$. The transformation $T$ as defined by equations (1) may be written in the form

$$
U=U(u, v), \quad V=V(u, v) .
$$

Of course each of the components $U=\phi+i \psi$ and $V=\phi-i \psi$ represents a polygenic function of the complex variable $u=x+i y$.

If the components $\phi$ and $\psi$ of (1) can be expressed as Taylor series of $(x, y)$, we shall say that each of the expressions $U$ and $V$ of $(5)$ is an analytic polygenic function. The set of holomorphic functions defining the conformal group is a proper subset of the class of analytic polygenic functions.

It can be shown that for any transformation $T$ defined by an analytic polygenic function, the right-hand sides of (5) can be expressed as Taylor series of $(u, v)$. Only for analytic transformations of this nature can we think of $u$ and $v$ as being independent complex variables, thus giving rise to the study of the four-dimensional complex plane. For any other transformation $T$, these minimal coordinates $(u, v)$ must be considered as conjugate complex variables.

3. The mean and phase derivatives. For any polygenic function $w=w(u, v)$, we consider the two linear differential operators

$$
\begin{aligned}
& w_{u}=\frac{\partial w}{\partial u}=\frac{1}{2}\left(\frac{\partial}{\partial x}-i \frac{\partial}{\partial y}\right) w=\frac{1}{2}\left(w_{x}-i w_{y}\right), \\
& w_{v}=\frac{\partial w}{\partial v}=\frac{1}{2}\left(\frac{\partial}{\partial x}+i \frac{\partial}{\partial y}\right) w=\frac{1}{2}\left(w_{x}+i w_{y}\right) .
\end{aligned}
$$

The operator $\partial / \partial u$ indicates performing the linear differential operation $(1 / 2)(\partial / \partial x-i \partial / \partial y)$, and is called the mean derivative. Similarly $\partial / \partial v$ means the application of the linear differential operation $(1 / 2)(\partial / \partial x+i \partial / \partial y)$, and is termed the phase derivative. In the case of analytic polygenic functions, these signify the formal partial derivatives with respect to $u$ and $v$.

The mean and phase derivatives are commutative. The Laplacian operator can be expressed as the product of these two operators except for a constant factor. That is 


$$
\begin{aligned}
w_{u v}=w_{v u}=\frac{\partial^{2} w}{\partial u \partial v}=\frac{\partial^{2} w}{\partial v \partial u} & =\frac{1}{4}\left(\frac{\partial^{2}}{\partial x^{2}}+\frac{\partial^{2}}{\partial y^{2}}\right) w \\
& =\frac{1}{4}\left(w_{x x}+w_{y y}\right) .
\end{aligned}
$$

By (6), it is found that the jacobian $J$ as given by (2) of any transformation $T$ (analytic or not) can be written in the form

$$
J=\phi_{x} \psi_{y}-\phi_{y} \psi_{x}=U_{u} V_{v}-U_{v} V_{u} \neq 0 .
$$

A harmonic transformation $T$ can be written in the form (5) where $U_{u v}=V_{u v}=0$; or in the more explicit form

$$
U=f(u)+g(v), \quad V=F(v)+G(u),
$$

where the four functions appearing on the right-hand sides are all analytic functions of one variable. In the real domain, the coefficients of $F$ and $G$ are the conjugates of those of $f$ and $g$ respectively.

By (8), it is found that the jacobian $J$ of a harmonic transformation (9) is

$$
J=f_{u} F_{v}-g_{v} G_{u} \neq 0 .
$$

By this, it can be shown that any pair of harmonic functions, one of which is not a linear integral function of the other with constant coefficients, defines a nondegenerate transformation of our harmonic set $(H)$.

4. Discussion of the harmonic transformation theory of certain isothermal families of curves. We shall study the problem of characterizing harmonic transformations by means of converting some isothermal families in the $(x, y)$-plane into isothermal families in the transformed $(X, Y)$-plane. Emphasis must be placed on the word some as we have proved elsewhere that the only point transformations carrying every isothermal family of curves into an isothermal family are the conformal ones.

It is evident by (9) that under any harmonic transformation $T$, a parallel pencil of straight lines in the $(X, Y)$-plane corresponds to an isothermal family of curves in the $(x, y)$-plane. We shall prove that this is characteristic of the infinite set $(H)$ of harmonic transformations, and of the products of conformal maps by circle-to-line transformations. The following precise result will be demonstrated.

TheOREM 1. If, by a transformation $T$ from the $(x, y)$-plane to the $(X, Y)$-plane, more than four distinct parallel pencils of straight lines 
in the $(X, Y)$-plane correspond to isothermal families in the $(x, y)$-plane, then in the real domain $T$ is a harmonic transformation or else $T$ is the product of a conformal map by a circle-to-line transformation; then necessarily every parallel pencil of straight lines in the $(X, Y)$-plane corresponds to an isothermal family in the $(x, y)$-plane.

It is remarked that a circle-to-line transformation is a point correspondence whereby every straight line in the transformed $(X, Y)$ plane corresponds to a circle or straight line in the $(x, y)$-plane. The circle-to-line transformations form an eleven-parameter set which includes the collineation group and the Moebius inversive group.

To discuss this result, we shall adopt minimal coordinates $(u, v)$. The transformation $T$, which is not necessarily an analytic polygenic function, can be written in the form (5) with nonvanishing jacobian $J$ as given by (8). Whenever $u$ (or $v$ ) appears as a subscript, the meaning is the application of the mean (or phase) derivative (6). Only where $T$ is defined by an analytic polygenic function will the subscripts denote formal partial differentiation with respect to $u$ (or $v$ ).

In minimal coordinates, a parallel pencil of straight lines in the $(X, Y)$-plane can be written in the form $V-m U=$ const., where $m$ is a fixed constant, essentially defining the direction of the parallel pencil. By the transformation $T$, this parallel pencil corresponds in the $(x, y)$-plane to the family of curves

$$
V(u, v)-m U(u, v)=\text { const. }
$$

For this family to be isothermal, the left-hand side must be a function of a harmonic function. If $\Delta$ denotes the expression

$$
\Delta=\frac{V_{u v}-m U_{u v}}{\left(V_{u}-m U_{u}\right)\left(V_{v}-m U_{v}\right)},
$$

the condition that (11) be isothermal is

$$
\Delta_{u} /\left(V_{u}-m U_{u}\right)=\Delta_{v} /\left(V_{v}-m U_{v}\right) .
$$

Substituting (12) into (13), this condition becomes

$$
\begin{gathered}
\left(V_{v}-m U_{v}\right)^{2}\left[\left(V_{u}-m U_{u}\right)\left(V_{u u v}-m U_{u u v}\right)\right. \\
\left.-\left(V_{u v}-m U_{u v}\right)\left(V_{u u}-m U_{u u}\right)\right] \\
=\left(V_{u}-m U_{u}\right)^{2}\left[\left(V_{v}-m U_{v}\right)\left(V_{u v v}-m U_{u v v}\right)\right. \\
\left.-\left(V_{u v}-m U_{u v}\right)\left(V_{v v}-m U_{v v}\right)\right] .
\end{gathered}
$$

Since this is of the fourth degree in $m$, it is obvious that, under a given transformation $T$, there can be at most four parallel pencils of straight lines in the $(X, Y)$-plane which correspond to isothermal families in the $(x, y)$-plane, unless (14) is an identity. 
Now let the transformation $T$ be such that there are more than four such distinct parallel pencils of straight lines in the $(X, Y)$-plane which correspond to isothermal families in the $(x, y)$-plane. Therefore the preceding equation is an identity in $m$.

Since the jacobian $J \neq 0$, it follows that at least one of the expressions $U_{u}$ or $U_{v}$ is not zero. Therefore the quantities $\left(V_{u}-m U_{u}\right)$ and $\left(V_{v}-m U_{v}\right)$ are relatively prime in the indeterminate $m$. Thus since each of these quantities are repeated factors of the identity (14), they must each be factors of the bracketed expressions in (14). The full discussion of the resulting identities is too complicated and will not be given here.

In conclusion, we find that in the imaginary domain the set of transformations

$$
\begin{array}{ll}
U=a(u) f(v)+h, & V=b(u) f(v)+k ; \\
U=a(v) f(u)+h, & V=b(v) f(u)+k ;
\end{array}
$$

where $(h, k)$ are constants, possess the property of Theorem 1 besides those there stated.

5. Discussion of harmonic maps of isothermal systems of curves. We shall consider those systems of curves which are converted into isothermal systems under harmonic transformations.

By appropriately changing the arbitrary constant to an isothermal parameter, it is known that any isothermal system of curves in the $(X, Y)$-plane can be written in the form

$$
F(U)+G(V)=\text { const. }
$$

We shall obtain a condition on $F$ and $G$ that this system shall correspond to an isothermal family in the $(x, y)$-plane under a harmonic transformation $T$.

Substituting the harmonic transformation $T: U=U(u, v)$, $V=V(u, v)$, where $U_{u v}=0$ and $V_{u v}=0$, into (16), we see that the corresponding family of curves in the $(x, y)$-plane is defined by $(16)$ also, in which the left-hand side is considered to be a function of $(u, v)$.

The condition that this system of curves in the $(x, y)$-plane be isothermal is

$$
\begin{aligned}
\left(F_{U} U_{v}\right. & \left.+G_{V} V_{v}\right)^{2}\left[\left(F_{U} U_{u}+G_{V} V_{u}\right)\left(F_{U U U} U_{u}^{2} U_{v}+G_{V V V} V_{u}^{2} V_{v}\right)\right. \\
& -\left(F_{U U} U_{u} U_{v}+G_{V V} V_{u} V_{v}\right)\left(F_{U U} U_{u}^{2}+G_{V V} V_{u}^{2}\right) \\
& \left.+\left(U_{u u} V_{u}-U_{u} V_{u u}\right)\left(F_{U U} G_{V} U_{v}-F_{U} G_{V V} V_{v}\right)\right] \\
= & \left(F_{U} U_{u}+G_{V} V_{u}\right)^{2}\left[\left(F_{U} U_{v}+G_{V} V_{v}\right)\left(F_{U U U} U_{u} U_{v}^{2}+G_{V V V} V_{u} V_{v}^{2}\right)\right. \\
& -\left(F_{U U} U_{u} U_{v}+G_{V V} V_{u} V_{v}\right)\left(F_{U U} U_{v}^{2}+G_{V V} V_{v}^{2}\right) \\
& \left.+\left(U_{v v} V_{v}-U_{v} V_{v v}\right)\left(F_{U V} G_{V} U_{u}-F_{U} G_{V V} V_{u}\right)\right] .
\end{aligned}
$$


THEOREM 2. The only isothermal systems of curves in the $(X, Y)$-plane which correspond to an isothermal family in the $(x, y)$-plane by every transformation of our harmonic set $(H)$ are the parallel pencils of straight lines.

This is deduced from (17) upon observing that it must be an identity in the partial derivatives of $U(u, v)$ and $V(u, v)$. Hence upon setting the coefficients of $U_{u u}, V_{u u}, U_{v v}, V_{v v}$ equal to zero, we find $F_{U U}=0$ and $G_{V V}=0$. Hence both $F$ and $G$ are linear integral functions of $U$ and $V$ respectively. Therefore the family in the $(X, Y)$-plane is a parallel pencil of straight lines.

6. Transformations whereby every pencil of straight lines corresponds to isothermal families. We wish to discuss in the real or imaginary domain the transformations whereby every pencil (parallel or not) of straight lines in the $(X, Y)$-plane corresponds to isothermal families of curves in the $(x, y)$-plane. Simultaneously we shall consider the corresponding problem with respect to every parallel pencil of straight lines and every set of concentric circles.

For this purpose, let $F=\log h, G=\epsilon \log k$ where $h=U-U_{0}$, $k=V-V_{0}$ and $\epsilon^{2}=1$. Thus (16) represents a pencil with finite vertex of straight lines or a concentric set of circles according as $\epsilon=-1$ or 1 .

Substituting these into (17) and simplifying the result, we obtain

$$
\begin{aligned}
\left(k U_{v}+\epsilon h V_{v}\right)^{2} & {\left[2\left(k U_{u}+\epsilon h V_{u}\right)\left(k^{3} U_{u}^{2} U_{v}+\epsilon h^{3} V_{u}^{2} V_{v}\right)\right.} \\
& -\left(k^{2} U_{u} U_{v}+\epsilon h^{2} V_{u} V_{v}\right)\left(k^{2} U_{u}^{2}+\epsilon h^{2} V_{u}^{2}\right) \\
& \left.-\epsilon\left(U_{u u} V_{u}-U_{u} V_{u u}\right)\left(k U_{v}-h V_{v}\right)\right] \\
= & \left(k U_{u}+\epsilon h V_{u}\right)^{2}\left[2\left(k U_{v}+\epsilon h V_{v}\right)\left(k^{3} U_{u} U_{v}^{2}+\epsilon h^{3} V_{u} V_{v}^{2}\right)\right. \\
& -\left(k^{2} U_{u} U_{v}+\epsilon h^{2} V_{u} V_{v}\right)\left(k^{2} U_{v}^{2}+\epsilon h^{2} V_{v}^{2}\right) \\
& \left.-\epsilon\left(U_{v v} V_{v}-U_{v} V_{v v}\right)\left(k U_{u}-h V_{u}\right)\right] .
\end{aligned}
$$

This is a polynomial of the sixth degree in $(h, k)$. We shall find all harmonic transformations for which this is an identity in $(h, k)$.

Upon setting the sixth degree terms equal to zero and simplifying, we find

$$
\begin{aligned}
(1+\epsilon)\left[k U_{u} U_{v}\right. & \left\{k\left(U_{u} V_{v}+U_{v} V_{u}\right)+2 h V_{u} V_{v}\right\} \\
& \left.-h V_{u} V_{v}\left\{2 k U_{u} U_{v}+h\left(U_{u} V_{v}+U_{v} V_{v}\right)\right\}\right]=0 .
\end{aligned}
$$

Upon setting the remaining terms of third degree in (18) equal to zero, we find 


$$
\begin{aligned}
& \left(k U_{v}+\epsilon h V_{v}\right)^{2}\left(k U_{v}-h V_{v}\right)\left(U_{u u} V_{u}-U_{u} V_{u u}\right) \\
& \quad=\left(k U_{u}+\epsilon h V_{u}\right)^{2}\left(k U_{u}-h V_{u}\right)\left(U_{v v} V_{v}-U_{v} V_{v v}\right) .
\end{aligned}
$$

THEOREM 3. The only transformations in the real or imaginary domain whereby every pencil of straight lines (parallel or not) in the $(X, Y)$-plane corresponds to an isothermal family of curves in the $(x, y)$ plane are the conformal maps, circle-to-line transformations, and products of conformal correspondences by circle-to-line transformations.

The set of harmonic transformations of Theorem 3 is

$$
U=a_{1} f(u)+b_{1} g(v), \quad V=a_{2} f(u)+b_{2} g(v),
$$

where $f$ and $g$ are analytic functions of $u$ and $v$ respectively with $f_{u} \neq 0$ and $g_{v} \neq 0$, and $\left(a_{1}, b_{1}, a_{2}, b_{2}\right)$ are complex constants such that $a_{1} b_{2}$ $-a_{2} b_{1} \neq 0$. In the real domain, the coefficients of $g$ are the conjugates of those of $f$, and $\left(b_{2}, a_{2}\right)$ are the conjugates of $\left(a_{1}, b_{1}\right)$ respectively.

To prove that (21) is the total set of harmonic transformations of Theorem 3, it is observed that $\epsilon=-1$ in the identity (18). It follows that (19) is satisfied identically. From (20), we deduce

$$
U_{u u} V_{u}-U_{u} V_{u u}=0, \quad U_{v v} V_{v}-U_{v} V_{v v}=0,
$$

since the jacobian $J$ of the transformation $T$ is not zero.

From these equations, we find

$$
\frac{U_{u}}{a_{1}}=\frac{V_{u}}{a_{2}}=f_{u}(u) \neq 0, \quad \frac{U_{v}}{b_{1}}=\frac{V_{v}}{b_{2}}=g_{v}(v) \neq 0,
$$

where $f$ and $g$ are analytic functions of $u$ and $v$ respectively, and $\left(a_{1}, b_{1}, a_{2}, b_{2}\right)$ are constants such that $a_{1} b_{2}-a_{2} b_{1} \neq 0$. Thus it follows that any harmonic transformation $T$ of Theorem 3 is a conformal map, or an affinity, or the product of a conformal correspondence by an affine transformation. That the harmonic transformation $T$ can be written in the form (21) follows from the fact that any affine transformation can be factored into the product of a translation by an affinity which leaves the origin fixed.

The validity of Theorem 3 is established as a consequence of Theorem 1 and the set (21).

7. Characterization of the conformal group. In this final section, we shall give a characterization of the conformal group in the real domain and also in the imaginary domain.

THEOREM 4. The only transformations in the real domain whereby every parallel pencil of straight lines and also every concentric set of 
circles in the $(X, Y)$-plane corresponds to an isothermal family of curves in the $(x, y)$-plane are those of the conformal group.

In the imaginary domain, the complete set of transformations possessing the property of Theorem 4 consists not only of conformalities but also of correspondences of the imaginary subset of harmonic transformation

$$
U=a_{1}[f(u)+g(v)], \quad V=a_{2}[f(u)-g(v)],
$$

where $f$ and $g$ are analytic functions of $u$ and $v$ separately with $f_{u} \neq 0$ and $g_{v} \neq 0$ and $\left(a_{1}, a_{2}\right)$ are complex constants such that $a_{1} \neq 0$ and $a_{2} \neq 0$. Any transformation of this set is the product of a conformal transformation followed by an affinity of the special form: $U=a_{1}(u+v), V=a_{2}(u-v)$.

The proof of the above result is as follows. By Theorem 1 , the required transformation $T$ is necessarily harmonic or else $T$ is the product of a conformal map by a circle-to-line transformation, or of the form (15). However, it can be shown that a circle-to-line transformation or one of the form (15) will possess the property of Theorem 4 if and only if it is conformal. Hence only harmonic transformations need be considered for which (18) must be an identity and $\epsilon=1$. From (19) and (20), we deduce the conditions

$$
\begin{aligned}
U_{u} U_{v}\left(U_{u} V_{v}+U_{v} V_{u}\right) & =0, & V_{u} V_{v}\left(U_{u} V_{v}+U_{v} V_{u}\right) & =0, \\
U_{u u} V_{u}-U_{u} V_{u u} & =0, & U_{v v} V_{v}-U_{v} V_{v v} & =0 .
\end{aligned}
$$

If $U_{u} V_{v}+U_{v} V_{u} \neq 0$, we find from these that our transformation $T$ is conformal. Otherwise the transformation $T$ must be necessarily of the form (21) where $U_{u} V_{v}+U_{v} V_{u}=0$. Hence $b_{1}=r a_{1}, b_{2}=-r a_{2}$, where $r \neq 0$. Upon replacing $g(v)$ by the new analytic function $g(v) / r$ we obtain our set (24). Of course, this set is imaginary, since for any real transformation $T$, the expression $\left(U_{u} V_{v}+U_{v} V_{u}\right)$ is always positive and can never be zero. This completes our discussion of Theorem 4 .

THEOREM 5. The only transformations in the real or imaginary domain which convert every pencil of circles into an isothermal family of curves are the conformal maps.

Let the pencils of circles be in the $(X, Y)$-plane. By Theorem 4 , the transformation $T$ must be either a conformality or a correspondence of the set (24). To prove our new result, it remains to show that, by any transformation of this set (24), not every pencil of circles in the $(X, Y)$-plane corresponds to an isothermal family of curves in the $(x, y)$-plane. 
Let us suppose that, by a transformation $T$ of the set (24), a parabolic pencil of circles $P_{0}$ in the $(X, Y)$-plane corresponds to an isothermal family in the $(x, y)$-plane. Now $T$ can be factored into the product of a conformal map $\Gamma$ by an affinity of the special form: $U=a_{1}(u+v), V=a_{2}(u-v)$. Since the inverse of $\Gamma$ carries the given isothermal family of curves into an isothermal family, it follows that, by this special affinity, the pencil $P_{0}$ must correspond to an isothermal family.

However, if the parabolic pencil of circles $P_{0}$ is taken to consist of all the circles tangent to the $Y$-axis at the origin, it is an easy matter to show that $P_{0}$ corresponds to a linear family of conic sections which is not isothermal, under this special affinity. Therefore, by any transformation $T$ of (24), the parabolic pencil of circles $P_{0}$ can not correspond to an isothermal family.

The preceding arguments show that the only transformations $T$ with the desired property are the conformalities. Theorem 5 is proved.

\section{BIBLIOGRAPHY}

The following papers constitute a short bibliography of the related subjects developed by Kasner and jointly by Kasner and DeCicco.

1. Conformal geometry, Proceedings of the Fifth International Congress of Mathematicians, vol. 2, p. 81, 1912.

2. The second derivative of a polygenic function, Trans. Amer. Math. Soc. vol. 30 (1928) pp. 803-818.

3. Lineal element transformations which preserve the isothermal character, Proc. Nat. Acad. Sci. U.S.A. vol. 27 (1941) pp. 406-409.

4. Generalized transformation theory of isothermal and dual families, Proc. Nat. Acad. Sci. U.S.A. vol. 28 (1942) pp. 52-55.

5. The geometry of polygenic functions, Revista de la Universidad de Tucuman vol. 4 (1944) pp. 7-45.

6. Generalized transformation theory of isothermal families, Revista de la Universidad de Tucumán vol. 4 (1944) pp. 91-94.

7. Geometry of the Fourier heat equation, Trans. Amer. Math. Soc. vol. 60 (1946) pp. 119-132.

8. Circle-to-line transformations, Amer. Math. Monthly vol. 52 (1945) pp. 425-433.

9. Theory of harmonic transformations, Proc. Nat. Acad. Sci. U.S.A. vol. 33 (1947) pp. 20-23.

Columbia University AND

ILLINOIS INSTITUTE OF TECHNOLOGY 